\title{
Proposing a New Strategy of Combining Machine Learning With Controlling The Cross-Transmission of Multidrug-Resistant Organisms in The ICU
}

\section{Zou Qian}

Shanghai Jiao Tong University School of Medicine

\section{Fei Kaihong}

Intensive Care Unit, Shanghai General Hospital

\section{Kang Mei}

Clinical Research Center, Shanghai General Hospital

\section{Li Xianchen}

Clinical Research Center, Shanghai General Hospital

\section{Ding Mengyuan}

School of Public Health, Shanghai Jiaotong University

\section{Geng Miaomiao}

School of Public Health, Shanghai Jiaotong University

Zhu Yanhong ( $\nabla$ yanhongzhu2010@163.com )

Shanghai Jiao Tong University School of Medicine

\section{Research article}

Keywords: multidrug-resistant, intensive care unit, cross-transmission, machine learning, precaution, prediction tool.

Posted Date: August 20th, 2020

DOI: https://doi.org/10.21203/rs.3.rs-56854/v1

License: (a) (1) This work is licensed under a Creative Commons Attribution 4.0 International License. Read Full License 


\section{Abstract}

Background: Multidrug-resistant organisms (MDROs) have become a global threat to public health. MDROs normally are transmitted from patient to patient via the hands of healthcare workers (HCWs). The key management of MDROs is control of dissemination as soon as possible.

Method: We established a bedside prediction tool based simply on experiences. According to the result of this predictive rule, we took a series of precautions in a general intensive care unit (ICU) from January 1 , 2018 , to December 31,2019 , in only one ward experimentally. We retrospectively analyzed medical records of patients between two wards to assess the efficiency of the routine care practice by performing different comparisons.

Results: A total of 636 patients were admitted in the ICU setting. After comparing two wards in hospital expenses and length of ICU stay, there were no significant differences. Precautions contributed to the association between room number and status of MDRO infection/colonization $(p=0.033)$, and the infection/colonization rate of MDROs was significantly different between the two wards $(p=0.006)$. The median of the length of clinical culture testing is 3.4 days.

Conclusion: Clinical culture testing is time-consuming. Advanced routine care practice controlled the cross-transmission of MDROs to some extent. This study highlights that a predicting model of MDROs is badly needed. Future researchers can engage in updating the predictive model focusing from machine learning-based risk algorithms, refer to experts' experiences and adopt more efficient precautions for strengthening the transmission efficiency.

\section{Background}

Multidrug-resistant organisms (MDROs) have become a global threat for public health. In America, more than 700,000 healthcare-associated infections occur annually, and many are caused by antibioticresistant bacteria(1). According to statistics from the China Antimicrobial Resistance Surveillance System (CARSS), methicillin-resistant Staphylococcus aureus (MRSAs) accounted for $30.9 \%$ of $S$. aureus isolates and carbapenem-resistant Acinetobacter baumannii accounted for $56.1 \%$ of baumannii isolates, respectively, in 2018(2). D.J. Morgan and Sarah S. Jackson found that when healthcare workers (HCWs) make contact with patients with MDR bacteria, they frequently contaminate their protective gowns and gloves $(3,4)$. Multidrug-resistant organisms normally are transmitted from patient to patient via the hands of HCWs(5). MDRO infection/colonization leads to clinical consequences, including intensive care unit (ICU) length of stay, limited treatment options, increased risk of therapeutic failure, and higher mortality and costs(6-9). The key step in decreasing the dissemination rate of MDROs is to cut off the spread chain from person to person.

In China, experts have advised that patients should have a positive screening once they are admitted to the ICU to determine whether they have colonized MDROs or not. This aims to take sequence precautions such as isolation to prevent the nosocomial cross-transmission of MDROs as early as possible, as 
isolation measures are recommended to reduce the transmission of $\operatorname{MDR}$ bacteria in the $\operatorname{ICU}(5,10)$. However, growing bacterial cultures and doing a susceptibility test take a few days in China. Usually, it is too late to benefit from isolation.

In this article, we introduce an exploratory experiment carried out in our hospital from January 1, 2018, to the present (March 2020). The experiment involves applying an elementary predictive model to prediscriminate the status of patients before the clinical culture testing results come out and then giving interventions first. We also evaluate the effect of the experiment in this article and offer some opinions on the management of MDROs in the ICU.

\section{Methods}

\section{Hospital setting}

This study was undertaken in a general ICU (including ward 2A and 2B) with 32 beds (2A has 12 beds) at a university-affiliated hospital located in Shanghai, China. It is a tertiary hospital, which is divided into a north and south branch. The experiment was completed in ward 2B of the south branch only because the author (Fei Kaihong) is the head nurse of the south branch's ICU (2B). Patients admitted to the ICU are assigned randomly to two wards. In 2B, there are 3 quadruple rooms (beds numbered from 11 to 14,19 to 22, and 23 to 26), 2 double rooms (beds numbered 15, 16, 17, and 18), and 6 single rooms.

\section{The exploratory experiment}

From January 1, 2018, to December 31, 2019, the head nurse of the 2B ward applied an elementary predictive rule to prediscriminate the status of MDROs colonization/infection. There are eight relevant factors in the predictive rule. If one clause is met, HCWs judged that the patient has a very high risk for colonization/infection-supposing to have colonized/infected MDROs. The details of this rule are shown in table 1. If the status is assumed to be colonized/infected with MDROs, the HCWs strengthen their hand hygiene and wear isolation gowns before and after contacting the patient. If circumstances permit, everyone predicted to be positive with MDROs should be isolated into a single room or infected or colonized patients should be separated from other patients. This preintervention continues until the clinical culture results are acquired.

\section{Table 1 An elementary predictive rule applied in ICU ward 2B}




\begin{tabular}{|l|}
\hline Clauses \\
\hline Antibiotic treatment before admission to the ICU \\
\hline Hospital stay longer than 2 weeks \\
\hline Underlying health problems and age older than 60 \\
\hline Recent invasive procedures \\
\hline Treatment with immunosuppressive agents \\
\hline Known colonization with MDROs \\
\hline Sepsis or organ failure before ICU admission \\
\hline Abrupt admission to the ICU \\
\hline
\end{tabular}

\section{Data collection}

Because this is a retrospective sharing of an ICU clinical practice, data were pulled from the hospital electronic file and CARSS retrospectively to assess the efficiency and benefit of this routine ICU care. Data included age, sex, ward (2A or 2B), hospitalization expenses (on April 1, 2020, 1 CNY=0.1408 \$USD), admission date, discharge data, length of hospital stay, bed number, and status of MDRO colonization/infection based on screening results from January 1, 2018, to December 31, 2019. To analyze the length of the clinical culture testing, we exported the date of sample submission and the date of test result acquisition from January 1, 2018, to December 31, 2019, of the whole ICU.

\section{Statistical analysis}

Quantitative variables that are normally distributed are expressed as the means (standard deviations); otherwise, they are expressed as medians (interquartile ranges). Categorical variables are described by number (percentage). Before the statistics, for quantitative variables, histograms and the Shapiro-Wilk test were used to assess the normality of the distribution. Quantitative variables were examined using Student's t-tests for normally distributed variables and the Wilcoxon rank-sum tests for nonnormally distributed variables. Chi-square tests were used for categorical variables. All reported P values are 2tailed, with a P-value $<0.05$ considered statistically significant. Statistical analyses were performed using IBM SPSS, 25.0.

We compared differences between 2A and 2B. Variables included age, sex, hospitalization expenses, ICU length of stay, and status of MDROs colonization/infection. We examined age as a categorical variable. Age categories used were $\geq 65$ years and $<65$ years. Hospitalization expenses were converted to US dollars (refer to the China-US exchange rate on April 1, 2020, 1 CNY=0.1408 \$). Hospitalization expenses were divided into two groups: $\geq 6500 \$$ and $<6500 \$$. Because patients in the ICU normally had tens of 
days of admission length, 14 days served as a cutoff point. ICU length of stay was examined as a categorical variable too.

\section{Ethical considerations}

The study was approved by the Shanghai General Hospital Institutional Review Boards. Written informed consent was not required.

\section{Results}

A total of 636 patients were admitted in the ICU setting. Of them, 198 came from $2 \mathrm{~A}$ and 438 came from 2B. The number of patients colonized/infected with MDROs was 68 , accounting for $10.69 \%$ of all specimens. Of the 636 patients, there were 389 males and 247 females. Their characteristics are summarized in Table 2.

\section{The exploratory experiment benefit evaluation}

Because the 2A ward did not have the experimental practices, we did performance comparisons between $2 \mathrm{~A}$ and $2 \mathrm{~B}$ in hospitalization expenses, ICU length of stay, and the real MDRO infection rate. The characteristics of the patients in the two wards and the performance comparison of the exploratory experiment are shown in table 2 . We can see that in the two wards, age and sex had no significant difference, $p=0.812$ and $p=0.212$, respectively. The performance comparison index of the exploratory experiment, for example, hospitalization expenses and ICU length of stay, had no significant difference either and were $p=0.219$ and $p=0.386$, respectively. However, there was a significant difference in MDRO infection status across these two wards' patients $(p=0.006,<0.05)$.

We also analyzed the association between room number and MDRO infection status. It is noteworthy that we excluded single rooms in this association analysis because they are prepared specifically for infected patients to isolate them from uninfected patients. There was a strong relevance between the single rooms and infected status-causing bias of our results. The results of the association are shown in table 3 . We could see that room number was associated with MDRO infection status, $p=0.033$. It suggested that the exploratory experimental routine practices may have truly discriminated against colonized/infected people and that the intervention curbed cross-transmission between rooms, so the infection occurred only in some specific rooms.

\section{Table 2 Characteristics of the patients of the two wards and performance comparison of the exploratory experiment}




\begin{tabular}{|c|c|c|c|c|}
\hline & \multicolumn{2}{|l|}{ Ward } & \multirow[t]{2}{*}{$x^{2}$} & \multirow[t]{2}{*}{$P$-value } \\
\hline & $2 \mathrm{~A}(\mathrm{~N} 0=$ & $2 \mathrm{~B}(\mathrm{~N} 1=438)$ & & \\
\hline Age & & & 0.057 & 0.812 \\
\hline$\geq 65$ & $72(36.4 \%)$ & $155(35.4 \%)$ & & \\
\hline$<65$ & $126(63.6 \%)$ & $283(64.6 \%)$ & & \\
\hline Sex & & & 1.558 & 0.212 \\
\hline male & $114(57.6 \%)$ & $275(62.8 \%)$ & & \\
\hline female & $84(42.4 \%)$ & $163(37.2 \%)$ & & \\
\hline Hospitalization expenses & & & 1.511 & 0.219 \\
\hline$\geq 6500, \$$ & $75(37.9 \%)$ & $144(32.9 \%)$ & & \\
\hline$<6500, \$$ & $123(62.1 \%)$ & $294(67.1 \%)$ & & \\
\hline ICU length of stay & & & 0.753 & 0.386 \\
\hline$\geq 14$, days, & $51(25.8 \%)$ & $99(22.6 \%)$ & & \\
\hline$<14$, days, & $147(74.2 \%)$ & $339(77.4 \%)$ & & \\
\hline MDRO infection status & & & 7.422 & 0.006 \\
\hline infected & $31(15.7 \%)$ & $37(8.4 \%)$ & & \\
\hline uninfected & $167(84.3 \%)$ & $401(91.6 \%)$ & & \\
\hline
\end{tabular}

Table 3 The association analysis between room number and MDRO infection status in ward 2B

\begin{tabular}{|c|c|c|c|c|}
\hline & \multicolumn{2}{|c|}{ MDRO infection status } & \multirow[t]{2}{*}{$\square^{2}$} & \multirow[t]{2}{*}{$P$-value } \\
\hline & infected & uninfected & & \\
\hline Room number & & & 10.472 & 0.033 \\
\hline 1 & $4(28.6 \%)$ & $54(20.9 \%)$ & & \\
\hline 2 & $6(42.9 \%)$ & $41(15.8 \%)$ & & \\
\hline 3 & $3(21.4 \%)$ & $49(19.0 \%)$ & & \\
\hline 4 & $0(0.0 \%)$ & $56(21.7 \%)$ & & \\
\hline 5 & $1(7.1 \%)$ & $58(22.5 \%)$ & & \\
\hline
\end{tabular}




\section{The length of clinical culture testing}

We exported the date of sample submission and subtracted the date of acquiring testing results to represent the length of clinical culture testing. From January 1, 2018, to December 31, 2019, we found 1065 ICU records in the hospital of the north branch. We found one outlier during statistical description. The data of clinical culture testing days did not conform to the normal distribution, $p<0.05$ (Shapiro-Wilk test). The median was 3.40 , and the interquartile range was 1.96 , with the outlier being excluded.

\section{Discussion}

To assess the efficiency of an index or risk score identifying MDRO status at hospital admission, the researchers used additional isolation days, costs of additional isolation, percentages of patients with ICUacquired infection of MDROs, ICU length of stay, ICU mortality, and calibration of a predictive model by the area under the curve (AUC)(11-14). In this study, we did not find significant differences between hospital expenses and length of ICU stay. The reason is that we analyzed the difference in age and sex between the two groups only since there were many factors associated with MDRO status, such as mechanical ventilation, chronic renal disease, indwelling catheter, previous hospital stay $\geq 7$ days, high APACHE-II score, and so on(15-18). A multicentre cohort study developed a prediction model with a good discrimination (AUC-ROC, $0.77 ; 95 \% \mathrm{Cl}, 0.72-0.82$ ), but the sensitivity was $67 \%$ and specificity $76.5 \%$, which could not be used to accurately identify patients(18). A study conducted in Brazil assessed the predictive model of carbapenem-resistant MDRGN acquisition in ICUs by AUC, yielding an acceptable result(19). Because it is a single-center study, we could not use that model in our hospital, and the situation is different between countries. A study launched in China came up with using American Thoracic Society Guidelines to predict the status of MDRO infection or colonization, but it failed(20). All these studies established a prediction model to avoid inappropriate empirical treatment clinically, but to a lesser extent in advanced intervention for MDROs transmission control.

In our study, there was an association between room number and status. To our knowledge, this is the first study to analyze the association between room number/bed number and MDRO infection status. The results suggested that the preintervention work and possibility of controlling cross-transmission of multidrug resistant pathogens. Discriminating infection or colonization is only the first step. The purpose is to control cross-transmission between patients. A study reported percentages of cross-transmission in ICU ranging from $23 \%$ to $53 \%$ due to patient contact(21). We know that colonized or infected patients, contaminated hands or gowns of HCWs, visitors, and environment all could be reservoirs of MDROs. A guideline suggested HCWs use contact precautions when providing care to patients who are known or suspected to be infected or colonized with MDROs(22). The key problem is knowing whether the patient has been infected or colonized as soon as possible. Guideline-recommended active surveillance contributes to stopping the spread of MDROs by early detection(10). N. Yamamoto found that rapid intervention based on a rapid molecular diagnostic assay contributed to reducing nosocomial transmission of carbapenem-resistant $A$. baumannii in ICUs(23). In this study, we analyzed the length of 
clinical culture testing, which took 3.4 days on average in our hospital. Therefore, we boldly speculated that active surveillance and molecular diagnostic assays are still time-consuming. A study validated previously reported clinical predictive algorithm to early identify the bacteria causing osteomyelitis but had a relatively poor diagnostic performance(24). In this article, we want to highlight the need for more machine-learning-based prediction model in medicine and the golden time for precaution is patient admission time.

The method of combining machine learning with MDRO status diagnosis in China is in the initial stage. We innovatively bring forward that the idea of using a predictive rule to prediscriminate the status of MDRO infection or colonization and then guiding accurate intervention on admission. Nevertheless, there are some limitations. First, we think the perfect endpoint of the benefit of precaution is the dissemination rate of MDRO. In our experiment, only patients identified by the predictive rule as MDRO infection/colonization were screened for the bacteria, so we could not calculate the cross-transmission rate and assess the predictive rule's discrimination and calibration. Second, the sample size is not big enough, and the mortality rate in the ICU is too small. Third, the baseline information is limited and cannot control the basic situations of patients in 2A and 2B. Moreover, the result of the comparison between the two groups lacked evidence. Most importantly, the predictive model was established empirically. In the future, we need to work on perfecting the new model with regard to both machine learning and consulting experts in nosocomial infection fields. To evaluate the new model, we should conduct a prospective cohort study or before-and-after study. Data on the baseline information of patients should be collected prospectively in as much detail as possible. Specific and effective precautions are eagerly figured out to advanced transmission control.

\section{Conclusion}

In this study, we found that the routine care practice, including predicting the status of MDROs and subsequent precautions, controlled the cross-transmission of MDROs to some extent. It sheds light on the notion that this is a feasible strategy for overcoming the challenge of controlling the transmission of nosocomial infection. Future researchers can engage in updating the predictive model based on big data, refer to experts' experiences and adopt more efficient precautions for strengthening the transmission efficiency.

\section{Declarations}

\section{Ethics approval and consent to participate}

The study was approved by the Shanghai General Hospital Institutional Review Boards. Written informed consent was not required. The procedures followed were in accordance with the ethical standards set by the institutional ethics committee and that of the Declaration of Helsinki.

\section{Consent for publication}


Not applicable.

\section{Availability of data and materials}

Authors can confirm that all relevant data are included in the article.

\section{Competing interests}

The author declare that they have no competing interests.

\section{Funding}

This work was supported by the General Program on National Natural Science Foundation of China (71974127) and Key Program on Hospital Management of China Hospital Development Institute of Shanghai JiaoTong University (CHDI-2018-A-04). The funder had role in the conception and design of this study and revising the manuscript critically for important intellectual content.

\section{Authors' contributions}

ZQ and FK contributed equally to the writing of this article. ZQ and FK were involved in the preparation of the proposal and study design and participated in data collection, data entry, and data analysis as well as manuscript preparation. KM and LX contributed to the acquisition of data, conception, and design of this study. DM and GM contributed to the acquisition, analysis, and interpretation of the data. ZY contributed to the conception and design of this study and was involved in revising the manuscript critically for important intellectual content. All authors read and approved the final manuscript.

\section{Acknowledgments}

We thank American Journal Experts (www.aje.cn) for its linguistic assistance during the preparation of this manuscript.

\section{Author's information}

Zou Qian, Ding Mengyuan, Geng Miaomiao (School of Public Health, Shanghai JiaoTong University, Shanghai, 200025, China). Fei Kaihong (Intensive Care Unit, Shanghai General Hospital, Shanghai, 201620, China). Kang Mei, Li Xianchen (Clinical Research Center, Shanghai General Hospital, Shanghai, 200080, China). Zhu Yanhong (Scientific Research Management Division, Shanghai General Hospital, Shanghai, 200080, China).

\section{References}

1. Magill SS, Edwards JR, Bamberg W, Beldavs ZG, Dumyati G, Kainer MA, et al. Multistate pointprevalence survey of health care-associated infections. N Engl J Med. 2014;370(13):1198-208. 
2. Committee of Experts on Rational Drug Use of the National Health and Family Planning Commission of the P.R.CHINA, China Antimicrobial Resistance Surveillance System. 2018 National Bacterial Resistance Surveillance Report. Chinese Journal of Rational Drug Use. 2020;17(01):1-10. doi:10. 3969/j.issn.2096-3327.2020.1.001

3. Morgan DJ, Rogawski E, Thom KA, Johnson JK, Perencevich EN, Shardell M, et al. Transfer of multidrug-resistant bacteria to healthcare workers' gloves and gowns after patient contact increases with environmental contamination. Crit Care Med. 2012;40(4):1045-51.

4. Jackson SS, Thom KA, Magder LS, Stafford KA, Johnson JK, Miller LG, et al. Patient contact is the main risk factor for vancomycin-resistant Enterococcus contamination of healthcare workers' gloves and gowns in the intensive care unit. Infect Control Hosp Epidemiol. 2018;39(9):1063-7.

5. Strich JR, Palmore TN. Preventing Transmission of Multidrug-Resistant Pathogens in the Intensive Care Unit. Infect Dis Clin North Am. 2017;31(3):535-50.

6. Roberts RR, Hota B, Ahmad I, Scott RD, 2nd, Foster SD, Abbasi F, et al. Hospital and societal costs of antimicrobial-resistant infections in a Chicago teaching hospital: implications for antibiotic stewardship. Clin Infect Dis. 2009;49(8):1175-84.

7. Melsen WG, Rovers MM, Groenwold RHH, Bergmans DCJJ, Camus C, Bauer TT, et al. Attributable mortality of ventilator-associated pneumonia: a meta-analysis of individual patient data from randomised prevention studies. The Lancet Infectious Diseases. 2013;13(8):665-71.

8. Bassetti M, De Waele JJ, Eggimann P, Garnacho-Montero J, Kahlmeter G, Menichetti F, et al. Preventive and therapeutic strategies in critically ill patients with highly resistant bacteria. Intensive Care Med. 2015;41(5):776-95.

9. Masse J, Elkalioubie A, Blazejewski C, Ledoux G, Wallet F, Poissy J, et al. Colonization pressure as a risk factor of ICU-acquired multidrug resistant bacteria: a prospective observational study. Eur J Clin Microbiol Infect Dis. 2017;36(5):797-805.

10. Tacconelli E, Cataldo MA, Dancer SJ, De Angelis G, Falcone M, Frank U, et al. ESCMID guidelines for the management of the infection control measures to reduce transmission of multidrug-resistant Gram-negative bacteria in hospitalized patients. Clin Microbiol Infect. 2014;20 Suppl 1:1-55.

11. Wassenberg M, Kluytmans J, Erdkamp S, Bosboom R, Buiting A, van Elzakker E, et al. Costs and benefits of rapid screening of methicillin-resistant Staphylococcus aureus carriage in intensive care units: a prospective multicenter study. Crit Care. 2012;16(1):R22.

12. Ledoux G, Six S, Lawson R, Labreuche J, Blazejewski C, Wallet F, et al. Impact of a targeted isolation strategy at intensive-care-unit-admission on intensive-care-unit-acquired infection related to multidrug-resistant bacteria: a prospective uncontrolled before-after study. Clin Microbiol Infect. 2016;22(10):888 e11- e18.

13. Djibre M, Fedun S, Le Guen P, Vimont S, Hafiani M, Fulgencio JP, et al. Universal versus targeted additional contact precautions for multidrug-resistant organism carriage for patients admitted to an intensive care unit. Am J Infect Control. 2017;45(7):728-34. 
14. Vasudevan A, Mukhopadhyay A, Li J, Yuen EG, Tambyah PA. A prediction tool for nosocomial multidrug Resistant Gram-Negative Bacilli infections in critically ill patients - prospective observational study. BMC Infect Dis. 2014;14:615.

15. Kiddee A, Assawatheptawee K, Na-Udom A, Boonsawang P, Treebupachatsakul P, Walsh TR, et al. Risk Factors for Extended-Spectrum beta-Lactamase-Producing Enterobacteriaceae Carriage in Patients Admitted to Intensive Care Unit in a Tertiary Care Hospital in Thailand. Microb Drug Resist. 2019;25(8):1182-90.

16. Carena AA, Laborde A, Roccia-Rossi I, Palacios CJ, Jordán R, Valledor A, et al. Proposal of a clinical score to stratify the risk of multidrug-resistant gram-negative rods bacteremia in cancer patients. The Brazilian journal of infectious diseases : an official publication of the Brazilian Society of Infectious Diseases. 2020;24(1):34-43.

17. Huang $H$, Chen B, Liu G, Ran J, Lian X, Huang X, et al. A multi-center study on the risk factors of infection caused by multi-drug resistant Acinetobacter baumannii. BMC infectious diseases. 2018;18(1):11.

18. Callejo-Torre F, Eiros Bouza JM, Olaechea Astigarraga P, Coma Del Corral MJ, Palomar Martínez M, Alvarez-Lerma F, et al. Risk factors for methicillin-resistant Staphylococcus aureus colonisation or infection in intensive care units and their reliability for predicting MRSA on ICU admission. Le infezioni in medicina. 2016;24(3):201-9.

19. Dantas LF, Dalmas B, Andrade RM, Hamacher S, Bozza FA. Predicting acquisition of carbapenemresistant Gram-negative pathogens in intensive care units. Journal of Hospital Infection. 2019;103(2):121-7.

20. Xie J, Ma X, Huang Y, Mo M, Guo F, Yang Y, et al. Value of American Thoracic Society guidelines in predicting infection or colonization with multidrug-resistant organisms in critically ill patients. PLoS One. 2014;9(3):e89687.

21. Lingaas $E$, Fagernes M. Development of a method to measure bacterial transfer from hands. J Hosp Infect. 2009;72(1):43-9.

22. Siegel JD, Rhinehart E, Jackson M, Chiarello L, Health Care Infection Control Practices Advisory C. 2007 Guideline for Isolation Precautions: Preventing Transmission of Infectious Agents in Health Care Settings. Am J Infect Control. 2007;35(10 Suppl 2):S65-164.

23. Yamamoto N, Hamaguchi S, Akeda Y, Santanirand P, Chaihongsa N, Sirichot S, et al. Rapid screening and early precautions for carbapenem-resistant Acinetobacter baumannii carriers decreased nosocomial transmission in hospital settings: a quasi-experimental study. Antimicrob Resist Infect Control. 2019;8:110.

24. Wade Shrader M, Nowlin M, Segal LS. Independent analysis of a clinical predictive algorithm to identify methicillin-resistant Staphylococcus aureus osteomyelitis in children. J Pediatr Orthop. 2013;33(7):759-62. 